\title{
Electrospun Nanofibers-Based Face Masks
}

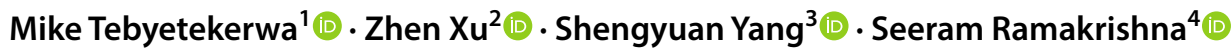

Received: 25 May 2020 / Accepted: 10 June 2020 / Published online: 25 June 2020

(c) Donghua University, Shanghai, China 2020

\begin{abstract}
Textiles have proved to be very important materials to human beings since the time immemorial. And, fibers are the basic building units of these materials. In this perspective we substantiate the uniqueness and capability of nanofibers as active layers in face masks, to protect people against the novel coronavirus disease (COVID-19). This time-sensitive letter introduces the mechanisms based on which their active filters function, the uniqueness of electrospun nanofibers in face masks and do-it-yourself (DIY) steps to realize a fully functional face mask at home.
\end{abstract}

Keywords Electrospinning $\cdot$ Nanofibers $\cdot$ Face masks $\cdot$ COVID-19 $\cdot$ Textiles

\section{Introduction}

Towards the end of December, 2019, an outbreak of a novel coronavirus disease (COVID-19) was reported in Wuhan, People's Republic of China [1]. As of 25 May 2020, the virus had spread to countries around the world and affected over 5.43 million people with more than 345,000 deaths reported [2]. To curb the rapid transmission of COVID-19,

Mike Tebyetekerwa

mike.tebyetekerwa@anu.edu.au

$\triangle$ Zhen Xu

z.xu19@imperial.ac.uk

$\triangle$ Shengyuan Yang

cmseysy@dhu.edu.cn

Seeram Ramakrishna

seeram@nus.edu.sg

1 Research School of Electrical, Energy and Materials Engineering, College of Engineering and Computer Science, The Australian National University, Canberra, ACT 2601, Australia

2 Department of Chemical Engineering, Imperial College London, London SW7 2AZ, UK

3 State Key Laboratory for Modification of Chemical Fibers and Polymer Materials, Shanghai "Belt \& Road" International Joint Laboratory for Advanced Fiber and Low-Dimension Materials, College of Materials Science and Engineering, Donghua University, Shanghai 201620, People's Republic of China

4 Centre for Nanofibers and Nanotechnology, National University of Singapore, Singapore 117581, Singapore various health organizations and responsible government ministries recommended people to use face masks to cover their nose and mouth when outside their homes. Moreover, the use of face mask is a standard safety procedure in some places and is a standard operating procedure for some workers, such as those in healthcare. The commonly used masks are the face (surgical) masks and respirator masks-P2 and N95 (Fig. 1a)[3]. Respirator masks are specially designed for high-risk medical settings and have the capability to protect the wearer from viruses and bacteria, whereas, surgical masks are designed for light medical settings and do not necessarily protect the wearer from bacteria and viruses. According to the world health organization, we quote that "Wearing a medical mask can limit the spread of certain respiratory viral diseases, including COVID-19. However, the use of a mask alone is not sufficient to provide an adequate level of protection. Other measures such as physical distancing and hand hygiene should be adopted" [4]. This makes it clear that a surgical mask alone cannot protect one from COVID-19 but can play some role in one's protection. This renders them important in the fight against COVID-19.

\section{How Face Masks Work?}

Face masks are made up of fine microscopic sieves which are the active layers driving the mask's working mechanism. The fine microscopic sieve is made up of entangled mats of very fine fibers capable of creating convoluted pathways that the air along with any particle or viruses and bacteria 
a

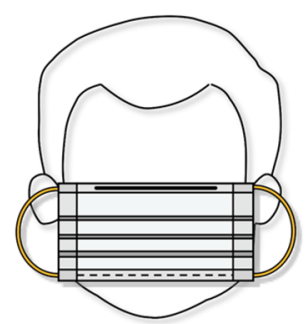

Face (Surgical) Masks

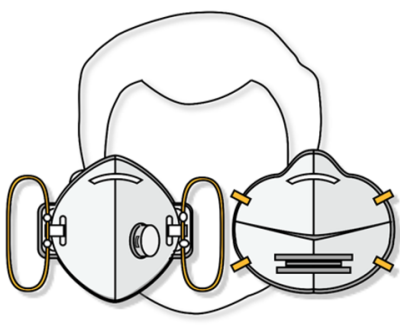

Respirator Masks - P2 \& N95 b

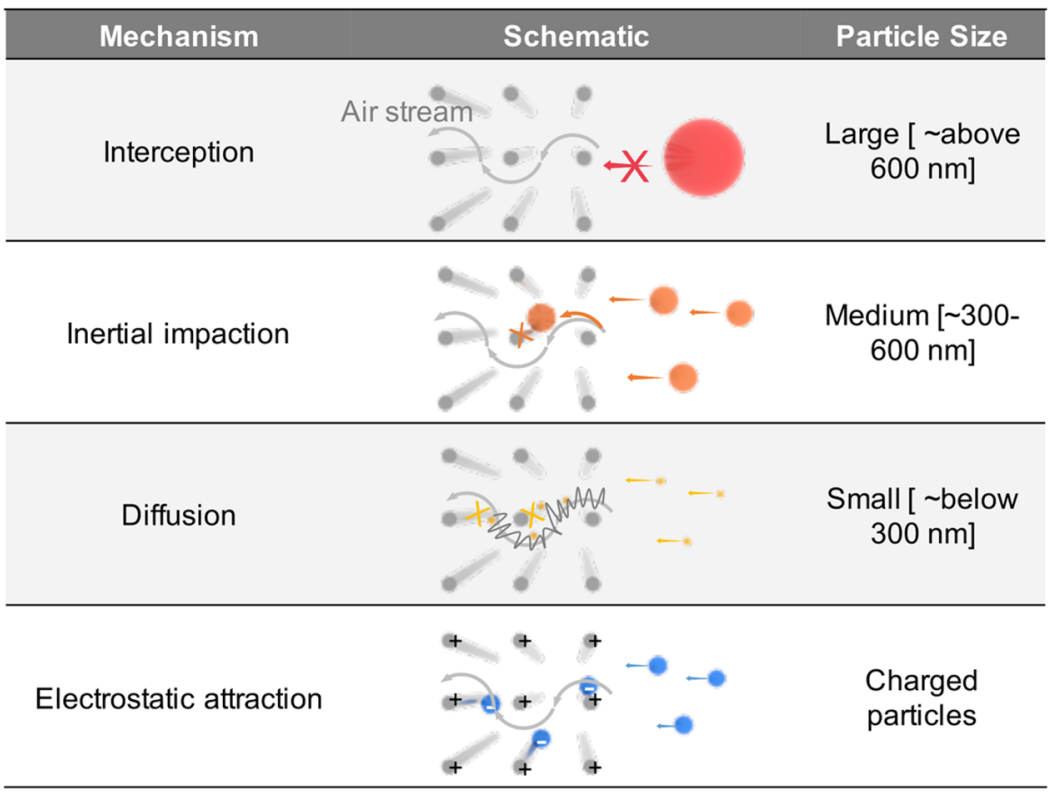

c
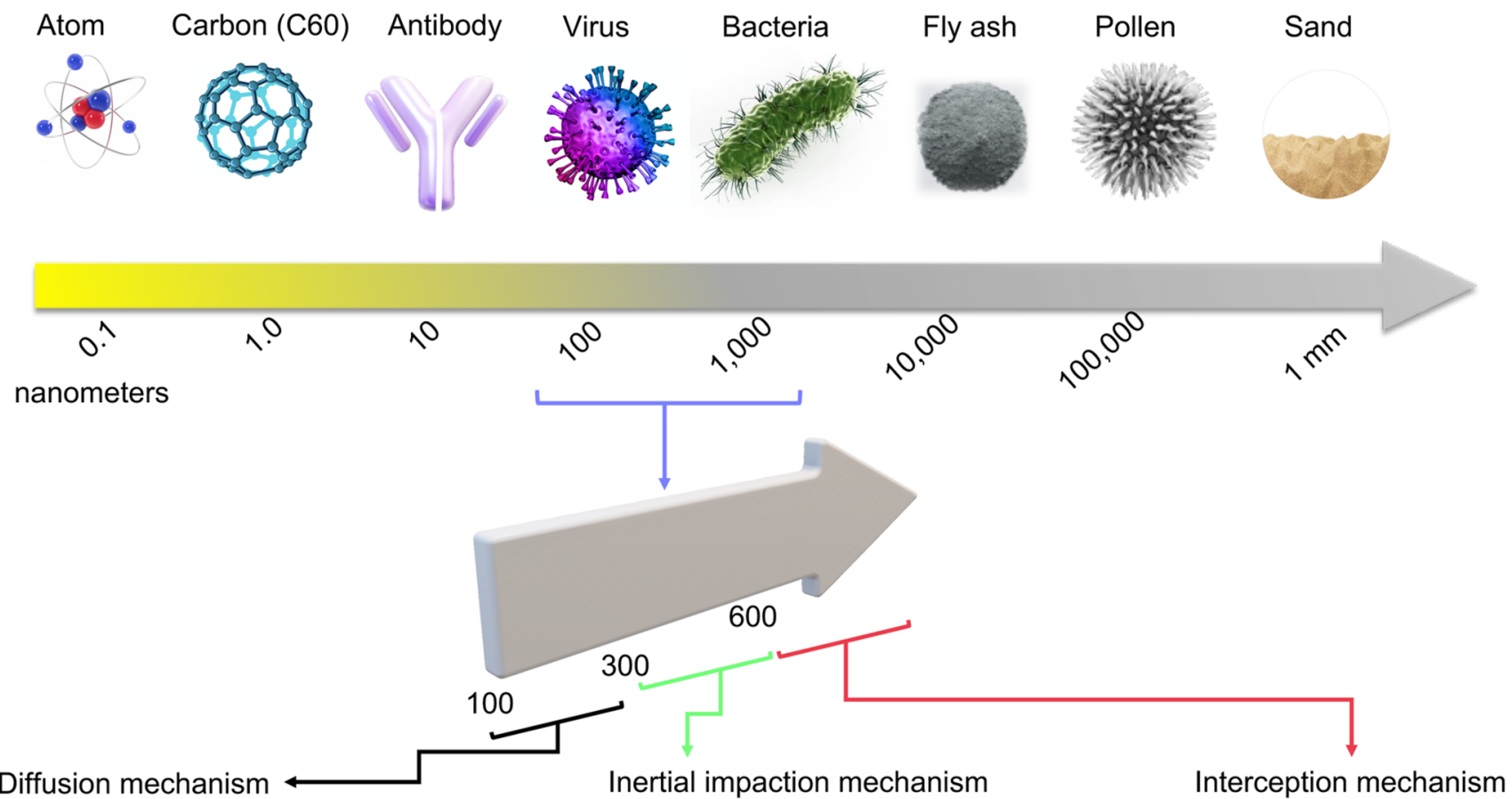

Fig. 1 a Common types of protective masks. b Mechanisms of performance of face masks. $\mathbf{c}$ Basic concept of size on length scale of various particles in nature and the mechanism associated with mask filters when filtering them

one inhales has to take when in use. When the face mask is in use, three different types of particles can be blocked from reaching the wearer in four separate mechanisms (Fig. 1b). Theses particles are separately classified in three sizes, macro, micro, and nano (Fig. 1c). Charged particles have also been added to the three particle types. Bigger macroparticles (above $600 \mathrm{~nm}$ ) usually with sizes above the pore sizes of the mats cannot be permitted through the filters and are immediately blocked outside the masks in a mechanism called the interception mechanism. For micro fine particles, 
$(\sim 300-600 \mathrm{~nm})$ these can possibly move through the mat pores of the mask sieve, but there is higher probability for them to crash (just like any object moving in a non-straight path at high velocities) on the fiber walls on their course through the fiber entanglements. This is highly dictated by the particles' mass and velocity, hence making the particle to never reach the wearer. This is called the impact/collision mechanism. For nano-sized particles (below $300 \mathrm{~nm}$ ), due to their very small sizes, they can easily move through the pores without even colliding with the pore walls with the help of air but are easily bombarded by the air molecules around them. For such particles to be captured, diffusionbased capture mechanism is followed which only happens in finer fibers (in nanometer sizes) and branched nanofibers. Electrospun nanofibers in this case prove more efficient. However, there are particles just between micro and nano ranges in a tune of $\sim 300 \mathrm{~nm}$, they hardly respect the impact/ collision mechanism and the diffusion-based capture mechanism, and thus prove hard to filter in many face masks. This, therefore, requires multiple layers of the mats to delay such particles and let them obey one of the mechanisms. The presence of multiple filtration layers creates new different engineering problem of breathability of the final product. It is therefore important for an engineer to address the two competing demands of air filterability and air breathability to meet the ideal performance of a face mask. Electrospun nanofibers provide the needed balance with right process control during electrospinning. The last mechanism is electrostatic driven filtration, where the filter is made up of charged mats which are capable of attracting the oppositely charged particles as a means of them not reaching the wearer.

\section{Uniqueness of Electrospun Nanofibers}

The porosity and surface area of the fibers in the face mask dictate their breathability and filterability. Thus, to overcome these two competing properties, one has to find ways to engineer and balance the pores in the active filtration layer as well as the surface area of the fibers making up the mat. With electrospinning (Fig. 2a), it is possible to control these two properties in situ during the preparation of the nanofibers. Porosity of the mats can be controlled by changing the spinning nozzles, polymer solutions and the collection geometry of the nanofibers, which rationally defines the resultant fiber diameters and their packing density [5]. Breathability of the filtration layer in the electrospun nanofibers can also be controlled in situ by controlling the fiber collection time, and collection geometry of the nanofibers during spinning which both dictate the final thickness of the mat and areal weight. The method of collection the nanofibers can result into random nonwoven mats, aligned or meshed structures (see examples in Fig. 2b). Each arrangement is known to provide special breathability factor.

Electrospun nanofibers exist as nonwoven mats welded together physically with their parent polymer joints. This is
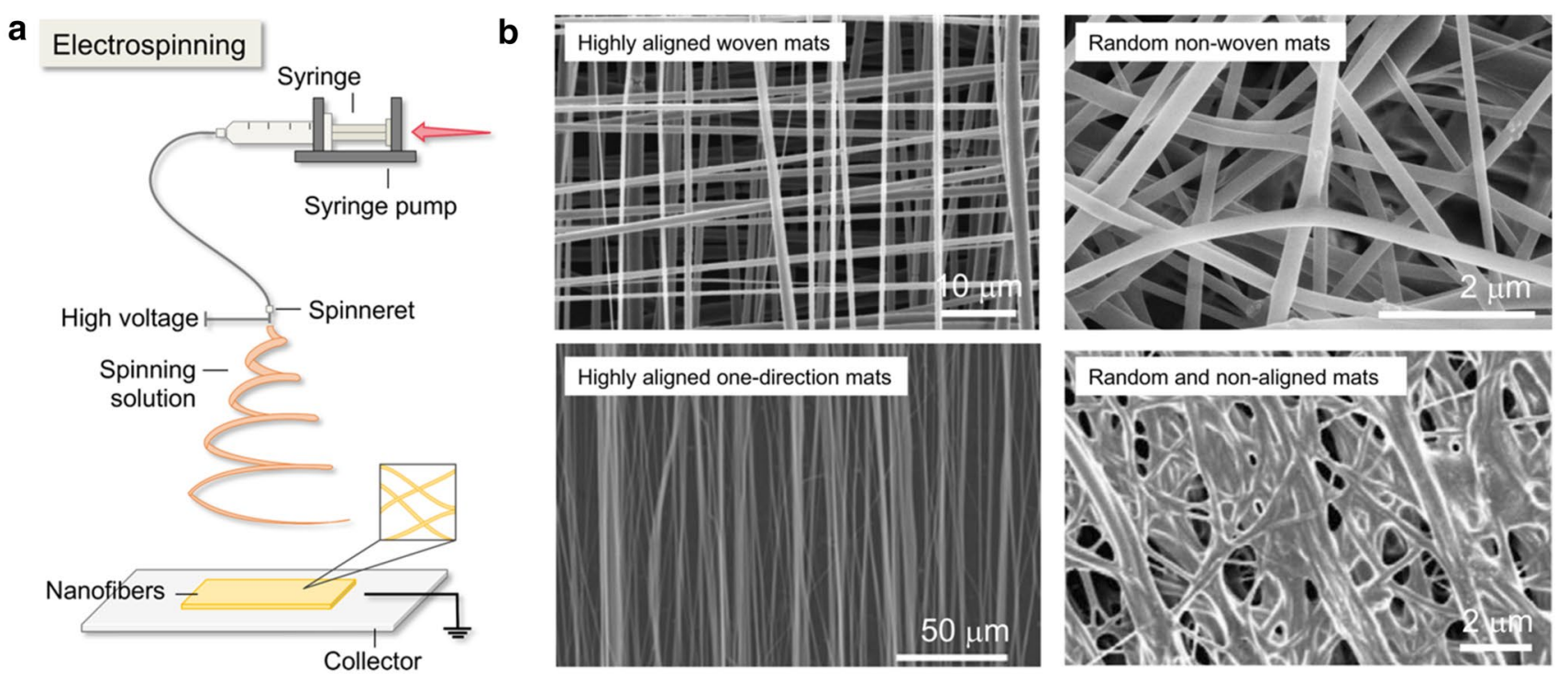

Fig. 2 a Electrospinning technique. b Scanning electron microscope images of electrospun nanofibers collected on different geometries and styles. Highly aligned nanofibers (Adapted and reproduced from https://www.nasa.gov/centers/langley/business/tg-img-fibermats. html,accessed May 23, 2020). Highly aligned one-directional mats [6]. Reproduced with permission. Copyright 2017 Royal Society of
Chemistry. Random nonwoven mats [7]. Reproduced with permission. Copyright 2018 Royal Society of Chemistry. Random nonwoven mats. Random and non-aligned mats [8]. Adapted and reproduced with permission. Copyright 2019 WILEY-VCH Verlag GmbH \& Co. KGaA, Weinheim 


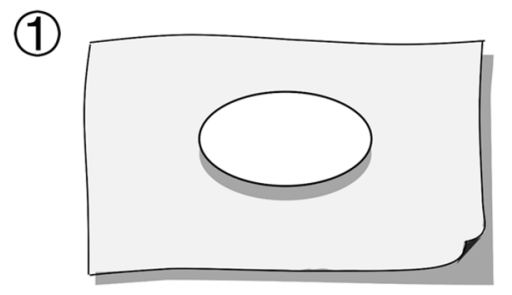

Cut a piece of fabric into suitable size and then place a piece of filter mat (electrospun nanofibers) at the middle of your fabric.
(2)

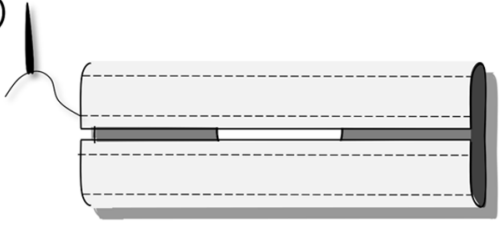

Fold the top and bottom parts of fabric to the middle, then stitch all the way cross
(3)

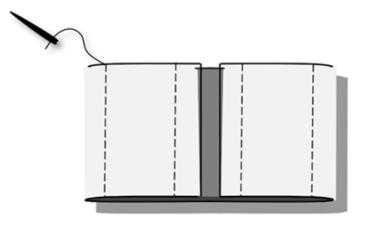

Fold the left and right parts of fabric to the middle, then stitch all the way cross as well, creating two hems.

\section{(4)}

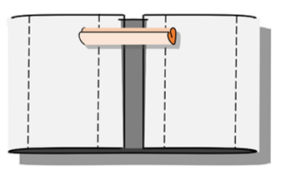

Stick a piece of rolled sponge at the top part of the fabric as nose pad.
(5)

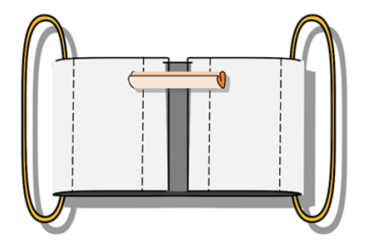

Thread an elastic through the two hems and then tie the ends of the elastic to make the ear loops.

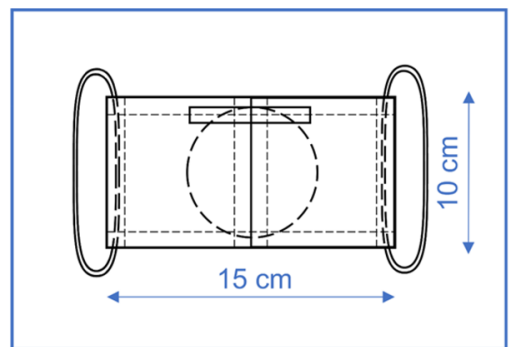

Structure diagram of the DIY finished face mask

Fig. 3 DIY steps of making a face mask at home

due to the fact that during the rapid electrospinning process in the high-field zone, the nanofibers continuously pile on each other when not perfectly dry, but in a wet state. Such a scenario makes the final nanofibers mats to have good physical interaction and durability during use. This is not the case in the common commercial face masks on market, whose active filter employs polypropylene (PP) fibers with small diameters in the range of $\sim 500-1000 \mathrm{~nm}$. The PP fibers filters achieves the three named filtration mechanisms with the fiber web kept together with the help of electrostatic charge responsible for keeping it in the required shape for maximum efficiency. Once the static charge is lost, their performance dramatically drops. Indeed, such filters lose static electricity when exposed to water and moisture, thus diminishing their filtering effect to almost half the original performance. Hence, the manufacturers recommend their disposal after a single use. Here, we propose to use electrospun non-woven fibers (with diameter below $100 \mathrm{~nm}$ ) as filter. These fibers as explained are physically bundled and welded onto each other in a durable web, thus overcoming the limitation of static loss mechanism-based filters. Thus, even in presence of fluids and moisture (if hydrophobic), and with the same porosity, these filters can maintain the same breathability as conventional PP filters while sustaining filtration efficiency of more of than $90 \%$.
To balance the two competing properties of breathability and filterability, therefore, electrospinning as a technique can be employed. With the right nozzle spinning gauges, collector geometry and polymer solution, it is possible to achieve balance between porosity and breathability of the mask filters at once in situ, thus making electrospinning technique favorable.

\section{Assembling Nanofibers-Based Face Mask At Home}

A wide range of portable, safe, battery-operated, and easyto-use electrospinners are available on the market which can be used to obtain the electrospun nanofiber mats with ease [9]. Then, after obtaining the right electrospun filter mats, they can be utilized when in a fully assembled working face mask. A do-it-yourself (DIY) approach to obtain a functional mask has been provided which can be followed at home (Fig. 3).

It is important to note that face mask should be made to conform to certain set standards. For example, breathability test MIL-M-36954 C: $\Delta \mathrm{P}[10]$-which quantifies the 
face mask's resistance to airflow, fluid resistance test ASTM F1862 [11]- which determines the resistance of the face mask to fluid penetration, particulate filtration test ASTM F2299 [12]- which evaluates the filterability of the face mask, bacterial filtration test ASTM F2101 [13]- which determines the amount of bacteria larger than $3000 \mathrm{~nm}$ that can possibly be filtered by the mask, flammability test 16 CFR Part 1610: flame spread [14]- which measures the flame resistance properties of the mask. Beyond these, other important regular tests can be carried out which include; biocidal efficiency, viricidal efficiency, skin sensitivity, allergy, toxicity, etc.

\section{Summary}

Until an effective vaccine is developed and made widely available, wearing protective face masks, maintaining personal hygiene and safe social distancing should be followed to prevent the spread of the COVID-19, and to provide a sense of security and well-being to everyone. This is leading to a worldwide surge in the use of billions of face masks every day-causing high demand for materials making them. In this work we have proposed the use of durable and yet reliable electrospun nonwoven filters with very small-fiber diameters (below $100 \mathrm{~nm}$ ). The filter can be processed by suitable disinfection methods and protocols to achieve reuse (increased usage time) without compromising the filtration efficiency. Beyond these, future face masks need to be antiviral as well as viricidal. The current electrospinning technology is mature [15], making the proposed strategy relatively low-cost with mass production capacity.

Acknowledgements M.T. acknowledges scholarship support from the Australian Government Research Training Program (RTP). Z.X. acknowledges the support from the China Scholarship Council (CSC). The Shanghai "Belt \& Road" International Joint Laboratory Program (18520750400) administrated by the Science and Technology Commission of Shanghai Municipality is also acknowledged for its support.

\section{Compliance with ethical standards}

Conflict of interest There is no conflict of interest to declare.

\section{References}

1. Wu F, Zhao S, Yu B, Chen Y-M, Wang W, Song Z-G, et al. A new coronavirus associated with human respiratory disease in China. Nature. 2020;579(7798):265-9.

2. Coronavirus disease (COVID-2019) situation reports. World Health Organisation (WHO). 2020. https://www.who.int/emerg encies/diseases/novel-coronavirus-2019/situation-reports. Accessed May 25, 2020.

3. Types and uses of face masks. 2020. https://www.safeworkau stralia.gov.au/doc/types-and-uses-face-masks. Accessed May 25, 2020.
4. Q\&A: Masks and COVID-19. 2020. https://www.who.int/emerg encies/diseases/novel-coronavirus-2019/question-and-answershub/q-a-detail/q-a-on-covid-19-and-masks. Accessed May 25, 2020.

5. Soliman S, Sant S, Nichol JW, Khabiry M, Traversa E, Khademhosseini A. Controlling the porosity of fibrous scaffolds by modulating the fiber diameter and packing density. J Biomed Mater Res A. 2011;96(3):566-74. https://doi.org/10.1002/jbm.a.33010.

6. Batnyam O, Suye S, Fujita S. Direct cryopreservation of adherent cells on an elastic nanofiber sheet featuring a low glass-transition temperature. RSC Adv. 2017;7(81):51264-71. https://doi. org/10.1039/c7ra10604a.

7. Marriam I, Wang XP, Tebyetekerwa M, Chen GY, Zabihi F, Pionteck J, et al. A bottom-up approach to design wearable and stretchable smart fibers with organic vapor sensing behaviors and energy storage properties. J Mater Chem A. 2018;6(28):13633-43. https ://doi.org/10.1039/c8ta03262a.

8. Li H, Wu T, Xue J, Ke Q, Xia Y. Transforming nanofiber mats into hierarchical scaffolds with graded changes in porosity and/or nanofiber alignment. Macromol Rapid Commun. 2020;41(3):e1900579. https://doi.org/10.1002/marc.201900579.

9. Portable Electrospinning. 2020. https://electrospintech.com/porta bleespin.html\#.XsvVgC2B1QJ. Accessed May 25, 20202020.

10. MIL-M-36954C. https://www.frazierinstrument.com/refer ence/standards/organizations/mil/abstract-mil-m-36954c.html (Accessed May 25, 2020).

11. ASTM F1862/F1862M-17, Standard test method for resistance of medical face masks to penetration by synthetic blood (Horizontal Projection of Fixed Volume at a Known Velocity), ASTM International, West Conshohocken, PA, 2017, www.astm.org. Accessed 25 May 2020.

12. ASTM F2299 / F2299M-03(2017), Standard test method for determining the initial efficiency of materials used in medical face masks to penetration by particulates using latex spheres, ASTM International, West Conshohocken, PA, 2017, www.astm.org. Accessed 25 May 2020.

13. ASTM F2101-19, Standard Test Method for Evaluating the Bacterial Filtration Efficiency (BFE) of Medical Face Mask Materials, Using a Biological Aerosol of Staphylococcus aureus, ASTM International, West Conshohocken, PA, 2019, www.astm.org. Accessed 25 May 2020.

14. 6 CFR Part 1610. Code of Federal Regulations, Title 16, Chapter II, Consumer Product Safety Commission.Federal Register 2007: 603-620. 16 CFR Part 1610. Standard for the flammability of clothing textiles, proposedrules. Federal Register.2007;72(38):8844-8868

15. Tebyetekerwa M, Ramakrishna S. What Is Next for Electrospinning? Matter. 2020;2(2):279-83. https://doi.org/10.1016/j. matt.2020.01.004.

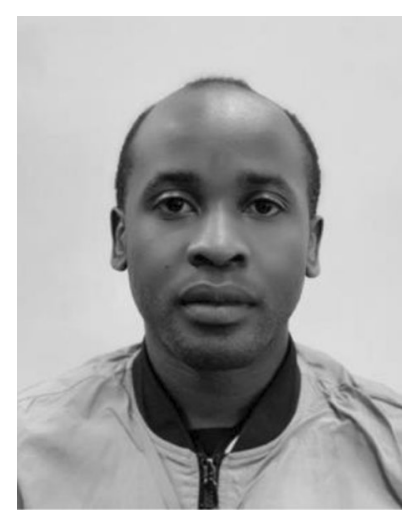

Mike Tebyetekerwa obtained his Masters in Materials Science and Engineering from Donghua University, Shanghai-China, in 2018 with his thesis focusing on functional fibrous materials under the co-supervision of Prof. Meifang Zhu and Dr Shengyuan Yang. He is currently a $\mathrm{PhD}$ candidate at the Australian National University, Canberra, with a focus on semiconducting 2D materials for use in future photovoltaics. His work focuses much on utilizing photoluminescence spectroscopy 
to understand these materials and their devices (including; silicon, perovskites, similar emerging and fluorescent polymer materials). He still carries out active research with regards to functional fibrous materials for energy and environment applications.

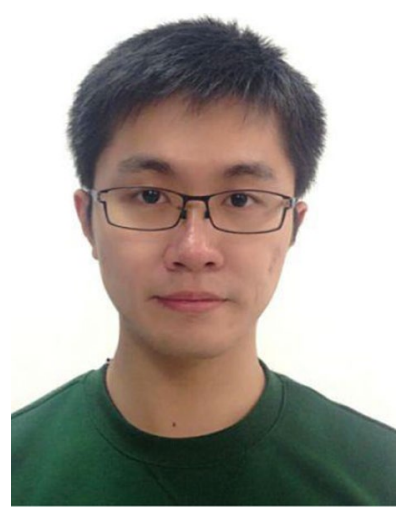

Zhen $\mathrm{Xu}$ received his Undergraduate degree (honours) in Polymer Materials and Engineering from Donghua University in 2017. His undergraduate research focused on electrospun Polyindole nanofibers for allsolid-state flexible supercapacitors under the supervision of Dr. Shengyuan Yang. He is currently a $\mathrm{PhD}$ candidate in Chemical Engineering at the Imperial College London, focusing on natureinspired hierarchical carbon materials for next-generation sodium batteries.

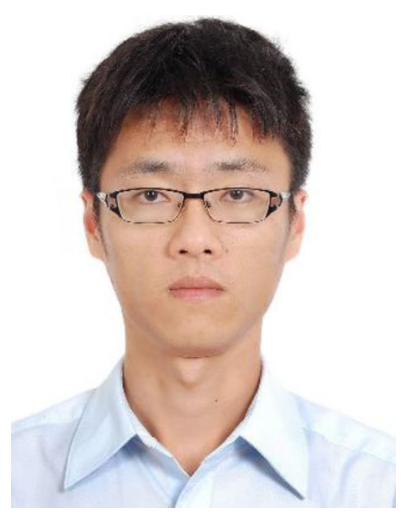

Dr Shengyuan Yang obtained his bachelor's degree from Fudan University in 2008. Then, he pursued his doctorate at the National University of Singapore under the supervision of Prof. Seeram Ramakrishna after receiving the prestigious NGS scholarship, which was completed in 2013. Finally, he returned home and joined the College of Materials Sciences and Engineering, Donghua University (DHU), in late 2014 as an associate professor. His research is centered on the novel applications of functional electrospun nanomaterials for wearable electronics and smart clothes. He is actively participating in several key national and state $R \& D$ projects. He is also currently the vice director of the DHU International Cooperation Office.

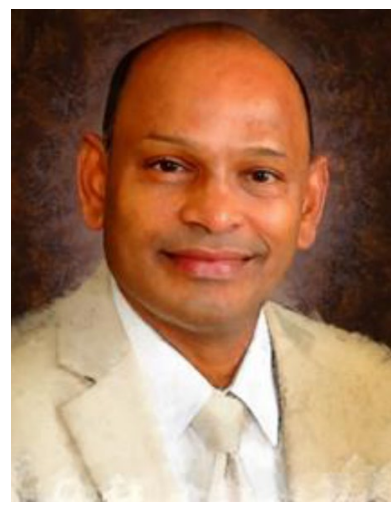

Academician Everest Chair Seeram Ramakrishna, FREng is a Professor at the National University of Singapore (NUS) at the center for nanofibers and nanotechnology. He received his $\mathrm{PhD}$ from the University of Cambridge, UK. He is an elected Fellow of UK Royal Academy of Engineering (FREng); Singapore Academy of Engineering; Indian National Academy of Engineering; and ASEAN Academy of Engineering \& Technology; American Association of the Advancement of Science (AAAS) and many others. Thomson Reuters identified him among the World's Most Influential Scientific Minds. His Google Scholar H-index exceeds 150 and his publications have attracted over 100,000 citations Clarivate Analytics recognized him among the Top 1\% Highly Cited Researchers in the world in materials science and crossfields categories. Microsoft Academic ranked him among the top 36 salient authors out of three million materials researchers worldwide. He innovatively utilizes electrospun nanofibers in various applications. 